formulaic training on good clinical practice - enabled the participation of hospitals already stretched by patients with COVID-19, with many of the less-research-experienced hospitals being among the best recruiters. Finally, a strong letter of support from the Chief Medical Officers of England, Wales, Scotland and Northern Ireland emphasized that the trial was to be seen as part of clinical care and stated that "Use of treatments outside of a trial, where participation was possible, is a wasted opportunity to create information that will benefit others."

The SOLIDARITY protocol was also a simple, pragmatic design adapted rapidly from a pre-existing core pandemic protocol. Multi-country involvement allowed recruitment to shift with disease incidence. A few countries, such as France (DisCoVeRy) - aided by the pre-existing REACTing network - started recruiting quickly, and within four weeks, seven countries were randomizing. Unfortunately, many others were delayed by approvals from national drug regulators, ethics committees or health ministries and missed the first wave's peak. Furthermore, countries lacked clear processes for prioritizing around larger pandemic trials, with many small trials hindering progress of the global mega-trial. Over-optimistic claims that fueled off-label use of drugs, such as hydroxychloroquine, also hindered recruitment.

While both trials' results are clinically important, the global research community can learn much from examining their processes. Countries should support clinical-trial networks that can quickly activate and adapt to contribute to large, simple multi-center trials that can study both older drugs and (if not unduly over-burdened with safety monitoring) new drugs, complementing more-detailed trials by helping assess any effects on mortality. Such large, simple, core studies should be national priorities, with coordinated support from chief medical officers, healthcare providers, funders and regulators to expedite set-up processes and promote rapid recruitment.

Rapid clear answers to treatment questions have the potential to save hundreds of thousands of lives during the course of the COVID-19 pandemic. In previous pandemics, large-scale randomized trials were generally not set up in time ${ }^{8,9}$. However, RECOVERY and SOLIDARITY have set new standards and have shown that a combination of old-fashioned randomization, established clinical-trials networks and imaginative use of modern information technology can provide many rapid and reliable therapeutic answers, following the recently published rationale for pursuing the magic of randomization rather than the myth of real-world evidence ${ }^{10}$. Throughout the world, however, over-regulation and the lack of national and international efforts to facilitate appropriately large trials represent a missed opportunity to improve care. During the COVID-19 pandemic, several false claims of efficacy have emerged from non-randomized comparisons (often misleadingly referred to as 'real-world evidence'), and it has been refreshing to see how perfectly such weakly founded claims can be swept aside by evidence from properly conducted, large-scale, randomized trials.

\section{Kari A. O. Tikkinen ${ }^{1,2}$, Reza Malekzadeh ${ }^{3}$, Martin Schlegel ${ }^{4}$, Jarno Rutanen ${ }^{5}$ and Paul Glasziou ${ }^{6}{ }^{4}$}

${ }^{1}$ Departments of Urology and Public Health, University of Helsinki and Helsinki University Hospital, Helsinki, Finland. ${ }^{2}$ Department of Surgery, South Karelian Central Hospital, Lappeenranta, Finland. ${ }^{3}$ Digestive Disease Research Institute, Tehran University of Medical Sciences, Tehran, Iran. ${ }^{4}$ Department of Anesthesiology and Intensive Care,
Technical University of Munich School of Medicine, Munich, Germany. ${ }^{5}$ Department of Internal Medicine, Tampere University Hospital, Tampere, Finland. ${ }^{6}$ Institute for Evidence-Based Healthcare, Bond University, Robina, Australia.

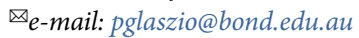

Published online: 22 September 2020 https://doi.org/10.1038/s41591-020-1077-z

References

1. Glasziou, P. P., Sanders, S. \& Hoffmann, T. Br. Med. J. 369, m1847 (2020).

2. The RECOVERY Collaborative Group. N. Engl. J. Med. https:// doi.org/10.1056/NEJMoa2021436 (2020).

3. Beigel, J.H. et al. N. Engl. J. Med. https://doi.org/10.1056/ NEJMoa2007764 (2020).

4. Yusuf, S., Collins, R. \& Peto, R. Stat. Med. 3, 409-422 (1984).

5. ISIS Collaborative Group. Lancet 2, 349-360 (1988).

6. Wise, J. \& Coombes, R. Br. Med. J. https://doi.org/10.1136/bmj. $\mathrm{m} 2670$ (2020).

7. Tenaerts, P., Madre, L. \& Landray, M. Clin. Trials 15, 5-12 (2018).

8. Muller, M. P., McGeer, A., Straus, S. E., Hawryluck, L. \& Gold, W. L. Emerg. Infect. Dis. 10, 389-394 (2004).

9. Tansey, C. M., Herridge, M. S., Heslegrave, R. J. \& Lavery, J. V. CMAJ 182, 1533-1537 (2010).

10. Collins, R., Bowman, L., Landray, M. \& Peto, R. N. Engl. J. Med. 382, 674-678 (2020).

Acknowledgements

We thank P. Horby and M. Landray (of RECOVERY), A.M. Henao-Restrepo, M.-P. Preziosi, J.-A. Røttingen and V. Sathiyamoorthy (of SOLIDARITY) and N. Peiffer-Smadja and Y. Yazdanpanah (of DisCoVeRy) for insightful advice. K.A.O.T. is supported by the Academy of Finland, Competitive Research Funding of the Helsinki and Uusimaa Hospital District and Sigrid Jusélius Foundation. The sponsors had no role in the analysis and interpretation of the data or the manuscript preparation, review, or approval.

\section{Author contributions}

K.A.O.T. and P.G. conceived of and drafted this Correspondence on the basis of interviews with trial investigators; all authors provided comments on the draft and approved the final version.

\section{Competing interests}

K.A.O.T. is the national principal investigator of the SOLIDARITY trial in Finland; R.M. is the national principal investigator of the SOLIDARITY trial in Iran; M.S. is a steering committee member of the SOLIDARITY trial in Germany; and J.R. is a steering committee member of the SOLIDARITY trial in Finland.

\title{
COVID-19 puts the Sustainable Development Goals center stage
}

To the Editor - A recent Comment ${ }^{1}$ and accompanying Editorial ${ }^{2}$ in Nature have raised the question of whether it is time to revise the United Nations (UN) Sustainable Development Goals (SDGs) and their various targets in the wake of the COVID-19 crisis. In their Comment, Naidoo and Fisher argue that it is "likely that many of the 169 targets will not be met by 2030 " and that "some could even be counterproductive" ${ }^{1}$. We argue for a more optimistic view and hold that the COVID-19 pandemic may help spur efforts to realize the UN's 2030 Agenda.

Rather than calling for a revision of the SDGs, COVID-19 should be seen as a catalyst for progress. COVID-19 has effectively unveiled the inequities and governance dysfunctions that the SDGs are poised to rectify ${ }^{3,4}$. By its very nature, a pandemic teaches that no one should be left behind and that no one is safe until everyone is safe.

Indeed, COVID-19 makes extant challenges impossible to ignore. It brings 
to the fore the consequences of not having health insurance or access to healthcare, not having water or a food supply during lockdown situations, or not having civil rights. With health as a lens, it is apparent how the myriad targets incorporated into the SDGs are interlinked and interdependent $t^{5}$.

As Naidoo and Fisher note, the

UN was founded to bring peace to a planet threatened by weapons that were far-reaching and dangerous. Viruses with pandemic potential are similarly far-reaching and dangerous. Now a supranational governance system is needed on par with that built 75 years ago, but this time the global challenge is to save future generations from the scourge of pandemics, not just from the scourge of war. Through efficient global coordination, medicines and vaccines must be able to be dispatched when an epidemic erupts, just as peace forces are called on when a conflict erupts. Security means transborder passage not only of troops but also of medicines and vaccines. From this perspective, the existing global governance system for health must not be allowed to implode. As pointed out by The LancetUniversity of Oslo Commission on Global Governance for Health ${ }^{6,7}$, the development of supranational governance organizations has not kept pace with the development of society at large. However, this 'stickiness' and other global governance dysfunctions $s^{6,7}$ call for refinement and modernization, not abandonment. The WHO and other important supranational organizations must be strengthened, not undermined.
What is needed is the development of a universal preparedness for health, in the spirit of the SDGs. While universal health coverage addresses present problems and challenges, universal preparedness for health takes the future as its yardstick. This implies an anticipatory approach to health: the root causes of zoonotic transmission must be investigated and vaccine strategies against zoonotic viruses must be developed before their pandemic potential is translated into a pandemic ${ }^{8}$. This time the world was caught unprepared the next pandemic virus should be given the shortest head start possible.

The SDGs instruct that universal preparedness for health requires a radical systems approach: health must be seen in the broadest of contexts, with due attention to social structures and infrastructure, working and living conditions ${ }^{3}$ and with strategies to counteract climate change, loss of biodiversity and human destruction of wild habitat ${ }^{9}$. In line with the overarching goal of leaving no one behind, preparedness means paying particular attention to vulnerable groups such as ethnic minorities, the elderly and people with underlying medical conditions. The 'oneness of humanity' thinking that so permeates the SDGs will stand to be tested once a vaccine against COVID-19 is at hand. A just and equitable distribution of affordable vaccines would represent a concrete and important step toward the realization of the SDGs.

Faced with the immense challenges embodied by the SDGs and accentuated by COVID-19, the last thing that one should do is to resort to pessimism or lower one's ambitions. The biggest threat to the implementation of the SDGs is resignation and indifference. The unique health challenges now being experienced should be used to leverage the implementation of the SDGs and help in navigation of "the forest of targets" that these goals embody. The SDGs are not up for revision but are more urgent and relevant than ever.

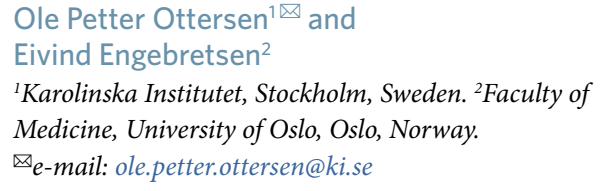

Published online: 9 October 2020

https://doi.org/10.1038/s41591-020-1094-y

References

1. Naidoo, R. \& Fisher, B. Nature 583, 198-201 (2020).

Nature 583, 331-332 (2020).

3. Bambra, C. et al. J. Epidemiol. Community Health https://doi org/10.1136/jech-2020-214401 (2020).

4. Sandset, T., Heggen, K. \& Engebretsen, E. Lancet 395, 1967 (2020).

5. Nilsson, M., Griggs, D. \& Visbeck, M. Nature 534, 320-322 (2016).

6. Ottersen, O. P., Frenk, J. \& Horton, R. Lancet 378, 1612-1613 (2011).

7. Engebretsen, E., Heggen, K. \& Ottersen, O. P. Lancet 389, 365 (2017).

8. El Zowalaty, M.E. \& Järhult, J.D. One Health https://doi.org/ 10.1016/j.onehlt.2020.100124 (2020)

9. Khetan, A. K. J. Gen. Intern. Med. 35, 2746-2747 (2020). 